\title{
Advance-Purchase Discounts as a Price Discrimination Device*
}

\author{
Volker Nocke ${ }^{\dagger}$ \\ University of Mannheim
}

\author{
Martin Peitz \\ University of Mannheim
}

\author{
Frank Rosar ${ }^{\S}$ \\ University of Bonn
}

First version: 19 May 2005

This version: 30 May 2010

\begin{abstract}
In an intertemporal setting in which individual uncertainty is resolved over time, advancepurchase discounts can serve to price discriminate between consumers with different expected valuations for the product. Consumers with a high expected valuation purchase the product before learning their actual valuation at the offered advance-purchase discount; consumers with a low expected valuation will wait and purchase the good at the regular price only in the event where their realized valuation is high. We characterize the profitmaximizing pricing strategy of the monopolist. Furthermore, adopting a mechanism design perspective, we provide a necessary and sufficient condition under which advance-purchase discounts implement the monopolist's optimal mechanism.
\end{abstract}

Keywords: advance-purchase discount, introductory offers, demand uncertainty, monopoly pricing, price discrimination, intertemporal pricing, mechanism design

JEL-Classification: L12, D42

${ }^{*}$ We would like to thank the Editors (Alessandro Lizzeri and Christian Hellwig) and three anonymous referees for helpful comments.

${ }^{\dagger}$ Department of Economics, University of Mannheim, 68131 Mannheim, Germany. Email: nocke@unimannheim.de. Also affiliated with CEPR, CESifo, and the University of Oxford.

${ }^{\ddagger}$ Department of Economics, University of Mannheim, 68131 Mannheim, Germany. Email: martin.peitz@googlemail.com. Also affiliated with CEPR, CESifo, ENCORE, and ZEW.

${ }^{\S}$ Department of Economics, University of Bonn, 53113 Bonn, Germany. Email: rosar@uni-bonn.de. 


\section{Introduction}

Advance-purchase discounts (introductory offers, early-booker discounts) have frequently been used in the sale of products such as holiday packages, hotel rooms, rental car hires, airline tickets, and conferences. While one may argue that, in these examples, a firm's pricing policy is affected by limited capacity, capacity constraints are largely absent in the digital economy where the use of advance-purchase discounts is also common. For example, amazon.com offers large discounts for pre-release orders of DVDs. Similarly, Apple's iTunes offers exclusive bonus tracks for pre-release orders of music albums. A theory of advance-purchase discounts that is applicable to the digital economy should therefore not rely on limited capacity. In this paper, we provide such a theory, based on price discrimination.

The starting point of our theory is the observation that consumers are likely to face uncertainty about their valuation when the time of consumption is far ahead in the future. Consider the following two-period problem of a monopolist selling a product with a fixed consumption or delivery date and committing to a price path. Consumers can buy either at the early date (before individual uncertainty is resolved) or at the late date (after individual uncertainty is resolved). At the early date, consumers only know their expected valuation. Suppose that all consumers have the same expected valuation (but differ in their ex-post valuations). By selling the good at the early date only ("advance selling"), the monopolist can extract all of the expected consumer surplus (namely, by charging an advance-selling price equal to consumers' common expected valuation). Such an advance-selling policy is clearly profit-maximizing, provided it is ex-post efficient to sell to all consumers. However, if the ex-post valuation of some consumers is lower than the unit cost of producing the good, it might be better for the monopolist to sell the good at the late date only ("spot selling"). While spot selling does not allow the monopolist to extract all of the consumer surplus (because of heterogeneity in ex-post valuations), it yields an ex-post efficient allocation conditional on a given level of output. As shown by Courty [3], the optimal pricing policy of the monopolist consists in advance selling if the unit cost of production is below a certain threshold and in spot selling otherwise. Advancepurchase discounts (where some consumers purchase at the early date and others at the late date) cannot be optimal in this simple setting because consumers are ex-ante identical.

In our model, consumers differ in their expected valuations. If the monopolist offers an advance-purchase discount (i.e., an increasing price path), consumers face a trade-off: they can either buy early at a discount or else wait and make their purchasing decision dependent on the actual realization of their valuation. Consumers with a high expected valuation will optimally purchase the good at the early date whereas consumers with a low expected valuation will buy the good at the late date (provided their ex-post valuation exceeds the price of the good at that date). That is, by offering an advance-purchase discount, the monopolist effectively discriminates between consumers on the basis of their expected valuations. ${ }^{1}$ We provide a necessary and sufficient condition under which the monopolist's optimal intertemporal pricing policy involves such an advance-purchase discount.

\footnotetext{
${ }^{1}$ For this discrimination to be effective, secondary markets should be closed down by the monopolist unless they can operate only at high costs for consumers. For instance, if the contract is personalized, a secondary market cannot be active.
} 
In the next section, we present our baseline model where the (binary) distribution of "shocks" to consumers' valuations is the same for all consumers, independently of their expected valuations. That is, we focus on consumer heterogeneity in expected valuations, abstracting from heterogeneity in the degree of uncertainty. In Section 3, we allow the monopolist to choose amongst simple pricing policies: advance selling (where all sales occur at the early date), spot selling (where all sales occur at the later date), and advance-purchase discounts (where some sales occur at the early date and some at the later date). We show that - even though all consumers face the same type of demand uncertainty - the monopolist may optimally use advance-purchase discounts. We provide a necessary and sufficient condition for this to be the case.

In Section 4, we consider an extended model where the shock to consumers' valuations is a function of the expected valuation. This allows us to analyze heterogeneity in the degree of uncertainty, while retaining the assumption that the distribution of consumer types is one-dimensional. In addition, we extend the model by introducing gradual resolution of uncertainty: consumers receive signals about their ex-post valuations at some intermediate date(s). This allows us to analyze whether the monopolist may want to further discriminate between consumers based on such signals. Adopting a mechanism design approach, we show that the optimal mechanism can always be implemented by a simple pricing policy at which sales are made only at an early date or at a late date (or both) but not at intermediate dates. We provide a necessary and sufficient condition for the optimality of each one of the three simple pricing policies (advance-purchase discounts, advance selling, and spot selling). In particular, advance-purchase discounts are optimal if consumer heterogeneity is sufficiently large; in that sense, the optimality of advance-purchase discounts emerges as the "normal" case.

Related Literature. Our paper contributes to the literature on intertemporal pricing. Previous work has identified a large class of economic environments in which, absent capacity costs, a monopolist will optimally sell all units at the same price (Riley and Zeckhauser [15]; Wilson [16]; Courty [3]). Advance-purchase discounts emerge as the optimal pricing policy in a number of economic environments where capacity is scarce and the aggregate level of demand is uncertain (Gale and Holmes [10,11], Dana $[6,7,8,9]$ ). Our paper is the first to show the optimality of advance-purchase discounts in a setting in which neither scarce capacity nor aggregate demand uncertainty play any role. ${ }^{2}$

Our paper is closely related to the analysis of refund contracts by Courty and Li [4]. However, the techniques of Courty and Li do not apply, in particular, because the distribution of shocks to consumer preferences is continuous in their model but discrete in ours. In Courty and $\mathrm{Li}$, before the individual uncertainty is resolved, consumers choose from a menu of contracts which may include fixed-price contracts and contracts with full or partial refunds. If a consumer has chosen a contract with full or partial refund, he has the option of returning the product for refund after learning his actual valuation. Courty and $\mathrm{Li}$ show that the optimal menu of contracts involves both full and partial refund contracts. ${ }^{3}$ In this paper, we focus in-

\footnotetext{
${ }^{2}$ In independent recent work, Möller and Watanabe [14] consider a model with two consumer types in which the seller can ex ante commit to her capacity. They show that advance-purchase discounts can be the preferred pricing policy if capacity costs are zero.

${ }^{3}$ Such refund contracts are, in effect, equivalent to option contracts, where a specified payment at the early
} 
stead on simple intertemporal pricing policies that are relevant in many real-world markets. In Courty and Li's setting, these simple intertemporal pricing policies correspond to menus that do no contain partial refund contracts. In real-world applications, simple pricing policies have an important advantage over contracts with partial or full refunds: a transaction takes place at one date only (the date of purchase) rather than at two dates (the date of contracting and the date at which the good is returned). ${ }^{4}$ We show that these simple pricing policies implement the optimal mechanism in a setting in which the monopolist can pick any, possibly stochastic, mechanism. Hence, the monopolist does not have an incentive in offering more sophisticated contracts even if they were available. However, as we discuss in the paper, this last result does rely on the assumed binary nature of information. In contrast to Courty and Li [4], we also allow for gradual resolution of uncertainty and show that the monopolist optimally refrains from using noisy information at some intermediate date.

Our paper is also related to the literature on monopoly bundling (see, e.g., McAfee, McMillan, and Whinston [13]) as one can interpret the consumption of the same good in different states as amounting to consuming different goods. By purchasing the good at the early date (before the preference shock is realized), a consumer effectively buys a consumption bundle, namely the right to consume the good in all states of nature. Advance-purchase discounts are, therefore, akin to mixed bundling.

Relating our paper to the literature on information acquisition (see, e.g., Cremer and Khalil [5], Lewis and Sappington [12], and, in particular, Bar-Isaac, Caruana and Cunat [2]), the price difference between the late and the early date can be interpreted as the information acquisition cost that a consumer has to incur so as to learn his willingness-to-pay. Hence, in our setting, the information acquisition cost is endogenous and controlled by the monopolist. In situations in which the monopolist optimally chooses advance-purchase discounts, the profit-maximizing information acquisition cost takes a strictly positive (and finite) value.

\section{$2 \quad$ A Simple Model}

Consider a monopolist who can choose to sell a product at two different dates, $t=0$ and $t=1$, at prices $p_{0} \in \mathbb{R}_{+} \cup\{\infty\}$ and $p_{1} \in \mathbb{R}_{+} \cup\{\infty\}$, respectively, and, prior to $t=0$, publicly commits to a price path $\left(p_{0}, p_{1}\right)$. We write $p_{t}=\infty$ if the monopolist does not make the product available at date $t$. The monopolist can produce any quantity of the good at constant marginal cost $c \geq 0$.

There is a unit mass of consumers with unit demand. A consumer is described by his type $r$, which denotes the expected valuation of the good. The consumer's ex-post valuation of the good is a random variable $v(r)$ with conditional expectation $\mathbf{E}[v(r) \mid r]=r$. The random variable $v(r)$ can take one of two values, a high value $v_{H}(r)$ with probability $\lambda_{H}$ and a low

date gives the consumer the right to exercise his option to buy the product at a predetermined price at the later date.

${ }^{4}$ Another advantage is that consumers contract sequentially under advance-purchase discounts. Thus, consumers who were initially not aware of the good can still purchase the good at a later date. Our formal analysis can be extended to allow for this possibility. 
value $v_{L}(r)<v_{H}(r)$ with the remaining probability $\lambda_{L}=1-\lambda_{H} \cdot{ }^{5}$ We assume $v_{z}(r)=r+\alpha_{z}$ for $z \in\{L, H\}$. That is, we assume here that the "shock" to the consumer's valuation, $\alpha_{z}$, is independent of the consumer's type $r$, where, by construction, the expected value of the shock is zero, $\lambda_{L} \alpha_{L}+\lambda_{H} \alpha_{H}=0$, and $\alpha_{H}>0>\alpha_{L}$. An alternative and equivalent interpretation is that the consumer derives a non-random utility from consumption of the good but that the value of the next-best alternative (the consumer's outside option) is uncertain. (Think of the chance of being invited to a party as an alternative to a concert for which you consider buying a ticket.)

We assume that the realization of the random variable is independent across consumers. That is, there is no aggregate uncertainty. At $t=0$, each consumer privately learns his own type $r$. At $t=1$, each consumer privately learns the realization of his valuation $v(r)$. Consumption takes place after $t=1$. If a consumer with valuation $v$ purchases the good (at either date) at price $p$, his (ex post) surplus from consuming the good is $v-p$. If he does not purchase the good, the surplus is zero.

Consumers differ in their expected valuation $r$. The cumulative distribution function over $r$ is denoted by $F: \mathbb{R}_{+} \rightarrow[0,1]$. We assume that $F$ is continuous on $\mathbb{R}_{+}$and has a density $f$ which takes values $f(r)>0$ for all $r \in(\underline{r}, \bar{r})$ and $f(r)=0$ for all $r \notin[\underline{r}, \bar{r}]$, and is continuous on $[\underline{r}, \bar{r}]$. Note that we allow for $f(\underline{r})$ and $f(\bar{r})$ to be zero or strictly positive. The upper bound of the support may be finite or infinite: $\bar{r} \in(\underline{r}, \infty) \cup\{\infty\}$. To avoid negative realizations, we assume that $\underline{r} \geq-\alpha_{L}$. Furthermore, the distribution of $r$ has a finite mean: $\int_{r}^{\bar{r}} r f(r) d r<\infty$.

Throughout this paper we make the monotone hazard rate assumption with respect to $F$.

Assumption The hazard rate $f(\cdot) /(1-F(\cdot))$ is strictly increasing in $r$ on the support of $F$.

The monotone hazard rate assumption is satisfied if and only if $1-F$ is strictly log-concave and holds for a variety of parametric distribution functions (see e.g. Bagnoli and Bergstrom [1]). Taking limits of the inverse hazard rate, we define

$$
\underline{\phi} \equiv \lim _{r \downarrow \underline{r}} \frac{1-F(r)}{f(r)} \text { and } \bar{\phi} \equiv \lim _{r \uparrow \bar{r}} \frac{1-F(r)}{f(r)} .
$$

Under the monotone hazard rate assumption, $\underline{\phi} \in \mathbb{R}_{+} \cup\{\infty\}$ and $\bar{\phi} \in \mathbb{R}_{+}$with $0 \leq \bar{\phi}<\underline{\phi}$. Note that $f(\bar{r})>0$ implies $\bar{\phi}=0$. To make the standard monopoly pricing problem at date $t=0$ well defined and non-trivial, we assume $\bar{r}>c+\bar{\phi}$.

\section{Optimal Intertemporal Price Discrimination}

The strategy of the monopolist is given by the price path $\left(p_{0}, p_{1}\right)$. Each price path is an element of one of three "classes" of simple pricing policies. First, an advance-purchase discount (APD) policy is defined by prices $p_{0}<p_{1}$ that induce strictly positive demand at both dates. Second,

\footnotetext{
${ }^{5}$ While the binary nature of uncertainty may feel special, consumers often face uncertainty of the yes/no-type in the real world. For example, the utility I get from attending a concert may greatly depend on whether or not my date shows up.
} 
an advance-selling policy is defined by an increasing price path $\left(p_{0}, p_{1}\right)$ with $p_{0}<p_{1}$ that induces positive demand at $t=0$ only. This advance-selling policy is payoff-equivalent to $\left(p_{0}, \infty\right)$, that is, the monopolist sells the product at $t=0$ only. Third, a spot-selling policy is defined by a decreasing price path, $\left(p_{0}, p_{1}\right)$ with $p_{0} \geq p_{1}$. A decreasing price path necessarily induces positive demand at $t=1$ only. This spot-selling policy is payoff-equivalent to $\left(\infty, p_{1}\right)$, that is, the monopolist sells the product at $t=1$ only.

In the following, we first characterize the profit-maximizing APD policy. We then compare this policy to the profit-maximizing advance-selling and spot-selling policies. Furthermore, we provide a necessary and sufficient condition under which the monopolist's optimal simple pricing policy involves advance-purchase discounts. Finally, we briefly discuss the welfare effects of prohibiting APD policies.

We first derive the monopolist's optimal APD policy. Suppose that consumers face an increasing price path $p_{0}<p_{1}$. If a consumer of type $r$ purchases the good at $t=0$, his expected surplus is $r-p_{0}$. Since the price at date 0 is lower than the price at date 1 , a rational consumer of type $r$ will delay his purchasing decision until $t=1$ only if he does not intend to buy the good in the event when $v(r)=v_{L}(r)$. If $r<p_{1}-\alpha_{H}$, the consumer will not buy the product even when $v(r)=v_{H}(r)$. If instead $r \geq p_{1}-\alpha_{H}$, the consumer will purchase the product at $t=1$ if and only if $v(r)=v_{H}(r)$, and so his expected surplus is $\lambda_{H}\left(r+\alpha_{H}-p_{1}\right)$. The gain in expected surplus from delaying the purchasing decision until $t=1$ is therefore $\Delta(r) \equiv \lambda_{H}\left(r+\alpha_{H}-p_{1}\right)-\left(r-p_{0}\right)$, which is strictly decreasing in $r$. Let $\widetilde{r}=\left[p_{0}-\lambda_{H}\left(p_{1}-\alpha_{H}\right)\right] /\left(1-\lambda_{H}\right)$ denote the consumer type who is indifferent between purchasing at $t=0$ and delaying the purchasing decision, i.e., $\Delta(\widetilde{r})=0$. If $r>\widetilde{r}$, the consumer will optimally purchase the product at $t=0$; if $r \in\left[p_{1}-\alpha_{H}, \widetilde{r}\right]$, he will purchase at $t=1$ if and only if $v(r)=v_{H}(r)$; otherwise, if $r<p_{1}-\alpha_{H}$, he will not buy at all. Thus, demand at date 0 is $1-F(\widetilde{r})$, while demand at date 1 is $\lambda_{H}\left[F(\widetilde{r})-F\left(p_{1}-\alpha_{H}\right)\right]$. The monopolist's profit is

$$
\pi\left(p_{0}, p_{1}\right)=\left(p_{0}-c\right)(1-F(\widetilde{r}))+\lambda_{H}\left(p_{1}-c\right) \begin{cases}F(\widetilde{r}) & \text { if } p_{1} \leq \underline{r}+\alpha_{H}, \\ {\left[F(\widetilde{r})-F\left(p_{1}-\alpha_{H}\right)\right]} & \text { otherwise. }\end{cases}
$$

We claim that any profit-maximizing price at date 1 satisfies $p_{1} \geq \underline{r}+\alpha_{H}$. To see this, suppose otherwise. But then the monopolist could increase her profit by raising both prices such that $\widetilde{r}$ (and, therefore, demand in each period) remains constant.

Lemma 1 Consider the set of prices $p_{0}$ and $p_{1}$ such that demand at both dates is positive, i.e., $p_{0}<p_{1}<\min \left\{p_{0}+\alpha_{H},\left[p_{0}-\left(1-\lambda_{H}\right) \underline{r}\right] / \lambda_{H}+\alpha_{H}\right\}$. If $\partial \pi\left(p_{0}, p_{1}\right) / \partial p_{0}<0$, then $\partial \pi\left(p_{0}^{\prime}, p_{1}\right) / \partial p_{0}<$ 0 for all $p_{0}^{\prime} \geq p_{0}$. That is, $\pi$ is quasi-concave in its first argument.

Proof. Note first that

$$
\frac{\partial \pi\left(p_{0}, p_{1}\right)}{\partial p_{0}}=1-F\left(\frac{p_{0}-\lambda_{H}\left(p_{1}-\alpha_{H}\right)}{1-\lambda_{H}}\right)-\left[\frac{p_{0}}{1-\lambda_{H}}-\frac{\lambda_{H} p_{1}}{1-\lambda_{H}}-c\right] f\left(\frac{p_{0}-\lambda_{H}\left(p_{1}-\alpha_{H}\right)}{1-\lambda_{H}}\right) .
$$


This expression is negative if and only if

$$
\left[\frac{p_{0}}{1-\lambda_{H}}-\frac{\lambda_{H} p_{1}}{1-\lambda_{H}}-c\right]\left\{\frac{f\left(\frac{p_{0}-\lambda_{H}\left(p_{1}-\alpha_{H}\right)}{1-\lambda_{H}}\right)}{1-F\left(\frac{p_{0}-\lambda_{H}\left(p_{1}-\alpha_{H}\right)}{1-\lambda_{H}}\right)}\right\}>1 .
$$

If $\partial \pi\left(p_{0}, p_{1}\right) / \partial p_{0}<0$, then the term in square brackets is positive and strictly increasing in $p_{0}$. The term in curly brackets is positive and, from the monotone hazard rate assumption, strictly increasing in $p_{0}$. Hence, if $\partial \pi\left(p_{0}, p_{1}\right) / \partial p_{0}<0$, then $\partial \pi\left(p_{0}^{\prime}, p_{1}\right) / \partial p_{0}<0$ for $p_{0}^{\prime} \geq p_{0}$.

The following lemma characterizes the unique candidate of the profit-maximizing APD policy $\left(\widehat{p}_{0}, \widehat{p}_{1}\right)$.

Lemma 2 If $c-\alpha_{H}>\underline{r}-\underline{\phi}$ the candidate for the profit-maximizing APD policy, $\left(\widehat{p}_{0}, \widehat{p}_{1}\right)$, is uniquely determined by

$$
\begin{aligned}
\widehat{p}_{1} & =c+\frac{1-F\left(\widehat{p}_{1}-\alpha_{H}\right)}{f\left(\widehat{p}_{1}-\alpha_{H}\right)} ; \\
\widehat{p}_{0} & =c+\lambda_{H} \frac{1-F\left(\widehat{p}_{1}-\alpha_{H}\right)}{f\left(\widehat{p}_{1}-\alpha_{H}\right)}+\left(1-\lambda_{H}\right)\left[\frac{1-F\left(\frac{\widehat{p}_{0}-\lambda_{H}\left(\widehat{p}_{1}-\alpha_{H}\right)}{1-\lambda_{H}}\right)}{f\left(\frac{\widehat{p}_{0}-\lambda_{H}\left(\widehat{p}_{1}-\alpha_{H}\right)}{1-\lambda_{H}}\right)}\right] .
\end{aligned}
$$

If $c-\alpha_{H} \leq \underline{r}-\underline{\phi}$ the candidate for the profit-maximizing APD policy, $\left(\widehat{p}_{0}, \widehat{p}_{1}\right)$, is uniquely determined by

$$
\begin{aligned}
& \widehat{p}_{1}=\underline{r}+\alpha_{H} \\
& \widehat{p}_{0}=\lambda_{H}\left(\underline{r}+\alpha_{H}\right)+\left(1-\lambda_{H}\right)\left[c+\frac{1-F\left(\frac{\widehat{p}_{0}-\lambda_{H} \underline{r}}{1-\lambda_{H}}\right)}{f\left(\frac{\widehat{p}_{0}-\lambda_{H} \underline{r}}{1-\lambda_{H}}\right)}\right] .
\end{aligned}
$$

In both cases, if the price path of the optimal pricing policy induces positive demand at each date, $\left(\widehat{p}_{0}, \widehat{p}_{1}\right)$ constitutes the optimal APD policy.

Proof. The two first-order conditions of profit maximization yield

$$
\widehat{p}_{0}-c=\lambda_{H}\left(\widehat{p}_{1}-c\right)+\left(1-\lambda_{H}\right)\left[\frac{1-F\left(\frac{\widehat{p}_{0}-\lambda_{H}\left(\widehat{p}_{1}-\alpha_{H}\right)}{1-\lambda_{H}}\right)}{f\left(\frac{\widehat{p}_{0}-\lambda_{H}\left(\widehat{p}_{1}-\alpha_{H}\right)}{1-\lambda_{H}}\right)}\right]
$$

and

$$
\widehat{p}_{1}-c=\frac{\left(\widehat{p}_{0}-c\right) f\left(\frac{\widehat{p}_{0}-\lambda_{H}\left(\widehat{p}_{1}-\alpha_{H}\right)}{1-\lambda_{H}}\right)+\left(1-\lambda_{H}\right)\left[F\left(\frac{\widehat{p}_{0}-\lambda_{H}\left(\widehat{p}_{1}-\alpha_{H}\right)}{1-\lambda_{H}}\right)-F\left(\widehat{p}_{1}-\alpha_{H}\right)\right]}{\lambda_{H} f\left(\frac{\widehat{p}_{0}-\lambda_{H}\left(\widehat{p}_{1}-\alpha_{H}\right)}{1-\lambda_{H}}\right)+\left(1-\lambda_{H}\right) f\left(\widehat{p}_{1}-\alpha_{H}\right)} .
$$

Inserting (8) into (9), and simplifying, we obtain (4). Recall that the optimal APD policy must be such that $\widehat{p}_{1} \in\left[\underline{r}+\alpha_{H}, \bar{r}+\alpha_{H}\right)$. Note that the LHS of (4) is strictly increasing in $\widehat{p}_{1}$, while 
(by the monotone hazard rate assumption) the RHS is strictly decreasing in $\widehat{p}_{1}$. Continuity then implies that if $\widehat{p}_{1} \in\left[\underline{r}+\alpha_{H}, \bar{r}+\alpha_{H}\right)$, it is uniquely determined by Eq. (4). Inserting (4) into (8) yields Eq. (5). By definition, an APD policy involves positive demand at both dates, i.e., $\widetilde{r}=\left[\widehat{p}_{0}-\lambda_{H}\left(\widehat{p}_{1}-\alpha_{H}\right)\right] /\left(1-\lambda_{H}\right) \in\left(\widehat{p}_{1}-\alpha_{H}, \bar{r}\right)$. The LHS of $(5)$ is strictly increasing in $\widehat{p}_{0}$, while (by the monotone hazard rate assumption) the RHS is strictly decreasing in $\widehat{p}_{0}$. Continuity then implies that if $\widetilde{r} \in\left(\widehat{p}_{1}-\alpha_{H}, \bar{r}\right), \widehat{p}_{0}$ is uniquely determined by Eq. (5). Note also that the monotone hazard rate assumption implies that $\widehat{p}_{0}<\widehat{p}_{1}$. To see this, suppose otherwise that $\widehat{p}_{0} \geq \widehat{p}_{1}$. From (4) and (5), it follows that

$$
\frac{1-F\left(\widehat{p}_{1}-\alpha_{H}\right)}{f\left(\widehat{p}_{1}-\alpha_{H}\right)} \leq \frac{1-F\left(\frac{\widehat{p}_{0}-\lambda_{H}\left(\widehat{p}_{1}-\alpha_{H}\right)}{1-\lambda_{H}}\right)}{f\left(\frac{\widehat{p}_{0}-\lambda_{H}\left(\widehat{p}_{1}-\alpha_{H}\right)}{1-\lambda_{H}}\right)} .
$$

From the monotone hazard rate assumption, $\widehat{p}_{1}-\alpha_{H} \geq\left[\widehat{p}_{0}-\lambda_{H}\left(\widehat{p}_{1}-\alpha_{H}\right)\right] /\left(1-\lambda_{H}\right)$, i.e., $\widehat{p}_{1} \geq \widehat{p}_{0}+\alpha_{H}$, contradicting that $\widehat{p}_{0} \geq \widehat{p}_{1}$.

The unique solution to (4) satisfies $\widehat{p}_{1}>\underline{r}+\alpha_{H}$ if and only if $c-\alpha_{H}>\underline{r}-\phi$. Otherwise, if $c-\alpha_{H} \leq \underline{r}-\underline{\phi}$, then $\widehat{p}_{1}$ must be given by the corner solution $\widehat{p}_{1}=\underline{r}+\alpha_{H}$. Substituting $\widehat{p}_{1}$ in (8) and rewriting yields $(7)$. The RHS of (7) is strictly increasing in $\widehat{p}_{0}$, while (by the monotone hazard rate assumption) the RHS is strictly decreasing in $\widehat{p}_{0}$ for $\widehat{p}_{0} \leq \lambda_{H} \underline{r}+\left(1-\lambda_{H}\right) \bar{r}$. If $\widetilde{r} \in(\underline{r}, \bar{r})$, continuity implies that $\widehat{p}_{0}$ is uniquely determined by $(7)$ and $\widehat{p}_{0}<\widehat{p}_{1} \cdot{ }^{6}$

It can easily be verified that the price path $\left(\widehat{p}_{0}, \widehat{p}_{1}\right)$ induces positive demand at both dates only if $c<\bar{r}-\alpha_{L}$, i.e., only if it is efficient to sell to the highest type(s) even when $v(r)=v_{L}(r)$.

We now turn to the characterization of the profit-maximizing advance-selling policy where, by definition, demand at $t=1$ is zero. Under such an advance-selling policy, the monopolist's profit can be written as

$$
\pi\left(p_{0}, \infty\right)=\left(p_{0}-c\right)(1-F(\widetilde{\widetilde{r}}))
$$

where $\widetilde{r}=p_{0}$ denotes the consumer type who is indifferent between purchasing at $t=0$ and not purchasing at all. Accordingly, the monopolist solves a standard monopoly problem with demand function $1-F(p)$. Let $p^{a} \equiv \arg \max _{p_{0}} \pi\left(p_{0}, \infty\right)$ denote the profit-maximizing advance-selling price.

Lemma 3 The profit-maximizing advance-selling price $p^{a}$ is uniquely determined by

$$
p^{a}=\left\{\begin{array}{cc}
c+\frac{1-F\left(p^{a}\right)}{f\left(p^{a}\right)} & \text { if } c>\underline{r}-\underline{\phi}, \\
\underline{r} & \text { otherwise. }
\end{array}\right.
$$

Proof. The first-order condition of profit maximization can be rewritten as

$$
\Psi\left(p_{0}\right) \equiv \frac{1}{f\left(p_{0}\right)} \frac{\partial \pi\left(p_{0}, \infty\right)}{\partial p_{0}}=\frac{1-F\left(p_{0}\right)}{f\left(p_{0}\right)}-\left(p_{0}-c\right)=0
$$

\footnotetext{
${ }^{6}$ We also note that, evaluated at $\widehat{p}_{0}=\underline{r}$, the RHS of (7) is greater or equal to the LHS since $c-\alpha_{H} \leq \underline{r}-\phi$. Note also that the RHS of (7) evaluated at $\widehat{p}_{0}=\underline{r}+\alpha_{H}$ is $\lambda_{H}\left[\underline{r}+\alpha_{H}-c\right]+\left(1-\lambda_{H}\right)\left[1-F\left(\underline{r}+\alpha_{H}\left(1-\bar{\lambda}_{H}\right)\right) \overline{]} / f(\underline{r}+\right.$ $\left.\alpha_{H}\left(1-\lambda_{H}\right)\right)$, which is smaller than $\underline{r}+\alpha_{H}-c$ if and only if $\underline{r}+\alpha_{H}>c+\left[1-F\left(\underline{r}+\alpha_{H}\left(1-\lambda_{H}\right)\right)\right] / f\left(\underline{r}+\alpha_{H}(1-\right.$ $\left.\lambda_{H}\right)$ ). Because of the monotone hazard rate assumption, this is implied by $\underline{r}+\alpha_{H}>c+\underline{\phi}$.
} 
Note that $\lim _{p_{0} \downarrow \underline{\underline{r}}} \Psi\left(p_{0}\right)>0$ if and only if $c>\underline{r}-\underline{\phi}$. Further, $\lim _{p_{0} \uparrow \bar{r}} \Psi\left(p_{0}\right)=\bar{\phi}-\bar{r}+c$, which is strictly negative by assumption. By the monotone hazard rate assumption, $\Psi$ is strictly decreasing in $p_{0} \in(\underline{r}, \bar{r})$. Hence, $p^{a}$ is uniquely determined by $\Psi\left(p^{a}\right)=0$ if $c>\underline{r}-\underline{\phi}$, and $p^{a}=\underline{r}$ otherwise.

Intuitively, the monopolist will prefer APD over advance selling (i) if marginal cost is high (since, in this case, it is inefficient to sell to low types when $z=L$ ) and (ii) if consumers face a large negative shock, i.e., $-\alpha_{L}$ is large (since, in this case, it becomes attractive for the monopolist to sell to low types only when $z=H$ ). The following lemma confirms this intuition.

Lemma 4 There exists an APD policy that yields strictly larger profits to the monopolist than the optimal advance-selling policy $\left(p^{a}, \infty\right)$ if and only if $\underline{r}-\underline{\phi}<c-\alpha_{L}$.

Proof. Suppose first that $c>\underline{r}-\phi$, so that $p^{a}>\underline{r}$. In this case, the profit from advance selling is equal to $\pi\left(p^{a}, p^{a}+\alpha_{H}\right)$. We show that $\lim _{p_{1} \uparrow p^{a}+\alpha_{H}}\left[\partial \pi\left(p^{a}, p_{1}\right) / \partial p_{1}\right]<0$ : starting from $p_{0}=p^{a}$ and $p_{1}=p^{a}+\alpha_{H}$ (where demand at $t=1$ is zero), a firm can increase its profit by marginally reducing its price at date 1 and thereby making sales at date 1 .

$$
\begin{aligned}
& \lim _{p_{1} \uparrow p^{a}+\sigma} \frac{\partial \pi\left(p^{a}, p_{1}\right)}{\partial p_{1}} \\
= & \left.\frac{d}{d p_{1}}\left[\left(p^{a}-c\right)\left[1-F\left(\frac{p^{a}-\lambda_{H}\left(p_{1}-\alpha_{H}\right)}{1-\lambda_{H}}\right)\right]\right]\right|_{p_{1}=p^{a}+\alpha_{H}} \\
& +\left.\frac{d}{d p_{1}}\left[\lambda_{H}\left(p_{1}-c\right)\left[F\left(\frac{p^{a}-\lambda_{H}\left(p_{1}-\alpha_{H}\right)}{1-\lambda_{H}}\right)-F\left(p_{1}-\alpha_{H}\right)\right]\right]\right|_{p_{1}=p^{a}+\alpha_{H}} \\
= & \left(p^{a}-c\right) \frac{\lambda_{H}}{1-\lambda_{H}} f\left(p^{a}\right)+\lambda_{H}\left(p^{a}+\alpha_{H}-c\right)\left[-\frac{\lambda_{H}}{1-\lambda_{H}} f\left(p^{a}\right)-f\left(p^{a}\right)\right] \\
= & -\alpha_{H} \frac{\lambda_{H}}{1-\lambda_{H}} f\left(p^{a}\right) \\
< & 0 .
\end{aligned}
$$

Suppose now that $c \leq \underline{r}-\underline{\phi}$, so that $p^{a}=\underline{r}$. Again, the profit from advance selling is equal to $\pi\left(p^{a}, p^{a}+\alpha_{H}\right)$, but now with $p^{a}=\underline{r}$. We show that $\lim _{p_{0} \downarrow \underline{r}}\left[\partial \pi\left(p_{0}, \underline{r}+\alpha_{H}\right) / \partial p_{0}\right]>0$ if and only if $c+\alpha_{H} \lambda_{H} /\left(1-\lambda_{H}\right)>\underline{r}-\underline{\phi}$ : starting from $p_{0}=\underline{r}$ and $p_{1}=\underline{r}+\alpha_{H}$ (where demand at $t=1$ is zero), by marginally increasing its price at date 0 (and thereby making sales at both dates), a firm can increase its profit if and only if $c+\alpha_{H}>\underline{r}-\underline{\phi}$. To see the "if" part, note that

$$
\lim _{p_{0} \downarrow \underline{\underline{r}}} \frac{\partial \pi\left(p_{0}, \underline{r}+\alpha_{H}\right)}{\partial p_{0}}=1-\left[\underline{r}-c-\frac{\lambda_{H}}{1-\lambda_{H}} \alpha_{H}\right] f(\underline{r}),
$$

which is positive if and only if $c-\alpha_{L}>\underline{r}-\phi\left(\right.$ since $-\alpha_{L}=\alpha_{H} \lambda_{H} /\left(1-\lambda_{H}\right)$ ). To see the "only if" part, note that the assumption that $c \leq \underline{r}-\phi$ implies $c-\alpha_{H}<\underline{r}-\phi$, and so the candidate for the optimal APD policy is such that $p_{1}^{-}=\underline{r}+\alpha_{H}$. Hence, this policy and the optimal advance-selling policy differ only in the price at date 0 . The assertion then follows from our earlier observation that $\pi$ is quasi-concave in $p_{0}$ for a fixed $p_{1}$ (Lemma 1): if $\lim _{p_{0} \downarrow \underline{r}}\left[\partial \pi\left(p_{0}, \underline{r}+\alpha_{H}\right) / \partial p_{0}\right]<0$, then $\partial \pi\left(p_{0}, \underline{r}+\alpha_{H}\right) / \partial p_{0}<0$ for all $p_{0} \geq \underline{r}$. 
Note that if $f(\underline{r})$ is sufficiently small so that $\phi$ is sufficiently large, there exists an APD policy that necessarily yields higher profits than àdvance selling.

We now turn to the characterization of the profit-maximizing spot-selling policy where, by definition, demand at $t=0$ is zero. Under such a spot-selling policy, the monopolist's profit can be written as

$$
\pi\left(\infty, p_{1}\right)=\lambda_{H}\left(p_{1}-c\right)\left[1-F\left(p_{1}-\alpha_{H}\right)\right]+\left(1-\lambda_{H}\right)\left(p_{1}-c\right)\left[1-F\left(p_{1}-\alpha_{L}\right)\right]
$$

Let $p^{s} \equiv \arg \max _{p_{1}} \pi\left(\infty, p_{1}\right)$ denote the profit-maximizing spot price.

Lemma 5 The profit-maximizing spot-selling price $p^{s}$ can be characterized as follows. If $p^{s} \geq$ $\bar{r}+\alpha_{L}$, the profit-maximizing spot price $p^{s}$ is uniquely determined by

$$
p^{s}= \begin{cases}c+\frac{1-F\left(p^{s}-\alpha_{H}\right)}{f\left(p^{s}-\alpha_{H}\right)} & \text { if } c-\alpha_{H}>\underline{r}-\underline{\phi} \\ \underline{r}+\alpha_{H} & \text { otherwise. }\end{cases}
$$

If $p^{s}<\bar{r}+\alpha_{L}$, the profit-maximizing spot price $p^{s}$ satisfies

$$
p^{s}=c+\frac{\left(1-\lambda_{H}\right)\left[1-F\left(p^{s}-\alpha_{L}\right)\right]+\lambda_{H}\left[1-F\left(p^{s}-\alpha_{H}\right)\right]}{\left(1-\lambda_{H}\right) f\left(p^{s}-\alpha_{L}\right)+\lambda_{H} f\left(p^{s}-\alpha_{H}\right)} .
$$

Proof. Suppose first that $p^{s} \geq \bar{r}+\alpha_{L}$, i.e., each consumer buys the good only when $v(r)=$ $v_{H}(r)$ if at all. In this case, the monopolist's profit can be rewritten as

$$
\pi\left(\infty, p_{1}\right)=\lambda_{H}\left(p_{1}-c\right)\left[1-F\left(p_{1}-\alpha_{H}\right)\right] .
$$

By the monotone hazard rate assumption, $\pi\left(\infty, p_{1}\right)$ is strictly quasi-concave in $p_{1}$. From the first-order condition, the profit-maximizing spot price $p^{s}$ satisfies

$$
p^{s}= \begin{cases}c+\frac{1-F\left(p^{s}-\alpha_{H}\right)}{f\left(p^{s}-\alpha_{H}\right)} & \text { if } c-\alpha_{H}>\underline{r}-\underline{\phi}, \\ \underline{r}+\alpha_{H} & \text { otherwise. }\end{cases}
$$

Suppose now that the spot price satisfies $p^{s}<\bar{r}+\alpha_{L}$ (i.e., there are some consumer types that consume independently of the realization of the shock). In that case, the monopolist's profit is given by

$$
\pi\left(\infty, p_{1}\right)=\left(p_{1}-c\right)\left\{\lambda_{H}\left[1-F\left(p_{1}-\alpha_{H}\right)\right]+\left(1-\lambda_{H}\right)\left[1-F\left(p_{1}-\alpha_{L}\right)\right]\right\},
$$

where $F\left(p_{1}-\alpha_{H}\right)=0$ and $f\left(p_{1}-\alpha_{H}\right)=0$ if $p_{1}<\underline{r}+\alpha_{H}$. From the first-order condition, the profit-maximizing spot price satisfies

$$
p^{s}=c+\frac{\left(1-\lambda_{H}\right)\left[1-F\left(p^{s}-\alpha_{L}\right)\right]+\lambda_{H}\left[1-F\left(p^{s}-\alpha_{H}\right)\right]}{\left(1-\lambda_{H}\right) f\left(p^{s}-\alpha_{L}\right)+\lambda_{H} f\left(p^{s}-\alpha_{H}\right)} .
$$

Note that, if $p^{s} \geq \bar{r}+\alpha_{L}$, the spot price coincides with the price at $t=1$ under the optimal APD policy, $p^{s}=\widehat{p}_{1}$, as can be seen from Eqs. (4) and (6). The following lemma shows that spot selling yields higher profits than advance-purchase discounts if and only if it is socially efficient to serve even a consumer of type $\bar{r}$ only when $v(r)=v_{H}(r)$. 
Lemma 6 There exists an APD policy that yields strictly larger profits to the monopolist than the optimal spot-selling policy $\left(\infty, p^{s}\right)$ if and only if $c-\alpha_{L}<\bar{r}-\bar{\phi}$.

Proof. Suppose first that $p^{s}<\bar{r}+\alpha_{L}$. Since $\pi\left(\infty, p^{s}\right)=\pi\left(p^{s}, p^{s}\right)$, spot selling is not optimal if

$$
\lim _{p_{0} \uparrow p^{s}} \partial \pi\left(p_{0}, p^{s}\right) / \partial p_{0}<0,
$$

i.e., if introducing a small advance-purchase discount, holding the date- 1 price fixed at $p^{s}$, increases the monopolist's profit. From (2) we have

$$
\lim _{p_{0} \uparrow p^{s}} \frac{\partial \pi\left(p_{0}, p^{s}\right)}{\partial p_{0}}=1-F\left(p^{s}-\alpha_{L}\right)-\left(p^{s}-c\right) f\left(p^{s}-\alpha_{L}\right) .
$$

This expression is negative if and only if

$$
p^{s}-c>\frac{1-F\left(p^{s}-\alpha_{L}\right)}{f\left(p^{s}-\alpha_{L}\right)} .
$$

Using (12), this inequality can be rewritten as

$$
\frac{\left(1-\lambda_{H}\right)\left[1-F\left(p^{s}-\alpha_{L}\right)\right]+\lambda_{H}\left[1-F\left(p^{s}-\alpha_{H}\right)\right]}{\left(1-\lambda_{H}\right) f\left(p^{s}-\alpha_{L}\right)+\lambda_{H} f\left(p^{s}-\alpha_{H}\right)}>\frac{1-F\left(p^{s}-\alpha_{L}\right)}{f\left(p^{s}-\alpha_{L}\right)},
$$

which is equivalent to

$$
\frac{1-F\left(p^{s}-\alpha_{H}\right)}{f\left(p^{s}-\alpha_{H}\right)}>\frac{1-F\left(p^{s}-\alpha_{L}\right)}{f\left(p^{s}-\alpha_{L}\right)} .
$$

This inequality is implied by the monotone hazard rate assumption.

Suppose second that $p^{s} \geq \bar{r}+\alpha_{L}$. We distinguish between two cases: (i) $\bar{r}-c+\alpha_{L}>\bar{\phi}$, and (ii) $\bar{r}-c+\alpha_{L} \leq \bar{\phi}$. We begin with case (i), $\bar{r}-c+\alpha_{L}>\bar{\phi}$. We show that, starting from any spot-selling policy $\left(\infty, p_{1}\right)$, the firm can increase its profit by selling to the highest types $\varepsilon$-close to $\bar{r}$ at date $t=0$ at price $\widetilde{p}_{0}=\left(1-\lambda_{H}\right)(\bar{r}-\varepsilon)+\lambda_{H}\left(p_{1}-\alpha_{H}\right)$ while holding the date-1 price fixed at $p_{1}$, provided $\varepsilon$ is sufficiently small. To see this, note that

$$
\begin{aligned}
\frac{\partial \pi\left(\widetilde{p}_{0}, p_{1}\right)}{\partial p_{0}} & =1-F(\bar{r}-\varepsilon)-\left[\bar{r}-\varepsilon-\frac{\lambda_{H} \alpha_{H}}{1-\lambda_{H}}-c\right] f(\bar{r}-\varepsilon) \\
& =1-F(\bar{r}-\varepsilon)-\left[\bar{r}-\varepsilon+\alpha_{L}-c\right] f(\bar{r}-\varepsilon) .
\end{aligned}
$$

Rearranging and taking the limit as $\varepsilon \rightarrow 0$, the RHS becomes negative if and only if

$$
\bar{r}+\alpha_{L}-c<\bar{\phi} \equiv \lim _{r \uparrow \bar{r}} \frac{1-F(r)}{f(r)},
$$

which holds by assumption in case (i). Hence, it cannot be optimal for the monopolist to sell at date $t=1$ only if $c-\alpha_{L}<\bar{r}-\bar{\phi}$.

We now turn to case (ii), $\bar{r}-c+\alpha_{L} \leq \bar{\phi}$. We show that, starting from any APD policy $\left(\widetilde{p}_{0}, \widetilde{p}_{1}\right)$ - which (by definition) induces positive demand at each date - the firm can increase its 
profit by slightly raising the date- 0 price while holding fixed the date- 1 price. From Eq. (3), we have $\partial \pi\left(\widetilde{p}_{0}, \widetilde{p}_{1}\right) / \partial p_{0}>0$ if and only if

$$
\widetilde{r}-c+\alpha_{L}<\frac{1-F(\widetilde{r})}{f(\widetilde{r})}
$$

where $\widetilde{r}=\left[p_{0}-\lambda_{H}\left(p_{1}-\alpha_{H}\right)\right] /\left(1-\lambda_{H}\right) \in(\underline{r}, \bar{r})$. The LHS is increasing in $\widetilde{r}$ while, by the monotone hazard rate assumption, the RHS is strictly decreasing in $\widetilde{r}$ and converges to $\bar{\phi}$ as $\widetilde{r} \rightarrow \bar{r}$. Hence the inequality is implied by $\bar{r}-c+\alpha_{L} \leq \bar{\phi}$, as postulated. By continuity of $\pi$, we thus have

$$
\pi\left(\widetilde{p}_{0}, \widetilde{p}_{1}\right)<\pi\left(\widetilde{p}_{1}, \widetilde{p}_{1}\right)=\pi\left(\infty, \widetilde{p}_{1}\right) \leq \pi\left(\infty, p^{s}\right),
$$

i.e., any APD policy yields strictly lower profits than the optimal spot-selling policy.

Using Lemmas 2, 4, and 6, we obtain the main result of this section.

Proposition 1 The monopolist's unique optimal simple pricing policy involves advance-purchase discounts, characterized in Lemma 2, if and only if $\underline{r}-\underline{\phi}<c-\alpha_{L}<\bar{r}-\bar{\phi}$.

Proof. By Lemma 4 there exist APD policies that yield higher profits than the profitmaximizing advance-selling policy if and only if $\underline{r}-\phi<c-\alpha_{L}$; by Lemma 6 there exist APD policies that yield higher profits than the profit-maximizing spot-selling policy if and only if $c-\alpha_{L}<\bar{r}-\bar{\phi}$. This implies that the optimal pricing policy must induce positive demand at each date. In this case the monopolist's optimal pricing policy is the APD policy $\left(\widehat{p}_{0}, \widehat{p}_{1}\right)$, which is characterized in Lemma 2 .

The proposition implies that, for advance-purchase discounts to be the monopolist's optimal pricing policy, there must be sufficient heterogeneity in the expected valuation $r$. In the limit as $\underline{r} \rightarrow-\alpha_{L}$ and $\bar{r} \rightarrow \infty$, the profit-maximizing pricing policy is necessarily an APD policy. In contrast, as heterogeneity in the expected valuation disappears, $\bar{r}-\underline{r} \rightarrow 0$, advance-purchase discounts are never optimal. ${ }^{7}$

We now briefly discuss the welfare consequences of prohibiting APD policies. We begin by providing a sufficient condition under which such a prohibition is undesirable.

Proposition 2 Suppose that $\underline{r}-\underline{\phi}<c-\alpha_{H}$ and $c-\alpha_{L}<\bar{r}-\bar{\phi}$, the inverse hazard function $[1-F(\cdot)] / f(\cdot)$ is weakly convex in $r$, and the optimal spot-selling price satisfies $p^{s} \geq \bar{r}+\alpha_{L}$. Then, prohibiting advance-purchase discounts reduces consumer surplus and total surplus (and makes no consumer better off).

Proof. Note that since $\underline{r}-\underline{\phi}<c-\alpha_{H}<c-\alpha_{L}<\bar{r}-\bar{\phi}$, by Proposition 1, the monopolist's optimal pricing policy involves advance purchase discounts. First, we compare the prices under an APD policy with the price under advance selling. We claim that consumers are better off under an APD policy than under advance selling, provided $c-\alpha_{H}>\underline{r}-\underline{\phi}$ and the inverse hazard function $[1-F(\cdot)] / f(\cdot)$ is weakly convex. To prove this claim, we first show that

\footnotetext{
${ }^{7}$ This confirms the finding by Courty [3] who considers the case where all consumers have the same expected valuation, which corresponds to $\underline{r}=\bar{r}$.
} 
$\widehat{p}_{1}<p^{a}+\alpha_{H}$. To see this, recall from Lemma 3 that the profit-maximizing advance-selling price (when $c-\alpha_{H}>\underline{r}-\underline{\phi}$ ) is given by

$$
p^{a}=c+\frac{1-F\left(p^{a}\right)}{f\left(p^{a}\right)} .
$$

Evaluating the RHS of this equation at $p^{a}=\widehat{p}_{1}-\alpha_{H}$ yields

$$
c+\frac{1-F\left(\widehat{p}_{1}-\alpha_{H}\right)}{f\left(\widehat{p}_{1}-\alpha_{H}\right)} .
$$

But, from Lemma 2, this expression is equal to $\widehat{p}_{1}$, which is larger than $p^{a}$ at $p^{a}=\widehat{p}_{1}-\alpha_{H}$. That is,

$$
p^{a}<c+\left.\frac{1-F(p)}{f(p)}\right|_{p^{a}=\widehat{p}_{1}-\alpha_{H}} .
$$

The LHS of the equation is increasing in $p^{a}$, while the RHS is decreasing in $p^{a}$ (by the monotone hazard rate assumption). Hence, there exists a unique $p^{a}>\widehat{p}_{1}-\alpha_{H}$ that satisfies Eq. (13). This means that all those consumers with types $\widehat{p}_{1}-\alpha_{H}<r<p^{a}-\alpha_{L}$ are strictly better off under APD than under advance selling.

From Lemma 2, the date-0 price of the profit-maximizing APD policy is given by

$$
\widehat{p}_{0}=\lambda_{H}\left[c+\frac{1-F\left(\widehat{p}_{1}-\alpha_{H}\right)}{f\left(\widehat{p}_{1}-\alpha_{H}\right)}\right]+\left(1-\lambda_{H}\right)\left[c+\frac{1-F\left(\frac{\widehat{p}_{0}-\lambda_{H}\left(\widehat{p}_{1}-\alpha_{H}\right)}{1-\lambda_{H}}\right)}{f\left(\frac{\widehat{p}_{0}-\lambda_{H}\left(\widehat{p}_{1}-\alpha_{H}\right)}{1-\lambda_{H}}\right)}\right] .
$$

We claim that $\widehat{p}_{0} \leq p^{a}$ if the inverse hazard function $[1-F(\cdot)] / f(\cdot)$ is weakly convex (with strict inequality under strict convexity). To see this, note that the RHS of Eq. (14) is a weighted average of $c+\left[1-F\left(r^{\prime}\right)\right] / f\left(r^{\prime}\right)$ and $c+\left[1-F\left(r^{\prime \prime}\right)\right] / f\left(r^{\prime \prime}\right)$ with weights $\lambda_{H}$ and $1-\lambda_{H}$, respectively, and with $r^{\prime}=\widehat{p}_{1}-\alpha_{H}$ and $r^{\prime \prime}=\left[\widehat{p}_{0}-\lambda_{H}\left(\widehat{p}_{1}-\alpha_{H}\right)\right] /\left(1-\lambda_{H}\right)$. The same weighted average of $r^{\prime}$ and $r^{\prime \prime}$ is equal to $\widehat{p}_{0}: \lambda_{H} r^{\prime}+\left(1-\lambda_{H}\right) r^{\prime \prime}=\widehat{p}_{0}$. If the inverse hazard function is linear (which is the case if the distribution of types is uniform), it follows that Eq. (14) can be rewritten as $\widehat{p}_{0}=c+\left[1-F\left(\widehat{p}_{0}\right)\right] / f\left(\widehat{p}_{0}\right)$. Comparing this equation with (13), we obtain that $\widehat{p}_{0}=p^{a}$. More generally, if the inverse hazard function is weakly convex, the RHS of Eq. (14) is weakly larger than $c+\left[1-F\left(\widehat{p}_{0}\right)\right] / f\left(\widehat{p}_{0}\right)$, so that $\widehat{p}_{0} \geq c+\left[1-F\left(\widehat{p}_{0}\right)\right] / f\left(\widehat{p}_{0}\right)$. By the monotone hazard rate assumption, there exists a unique $\widehat{p}_{0} \leq p^{a}$ that satisfies Eq. (14). This means that no consumer is worse off under APD than under advance selling.

Second, we compare the prices under an APD policy to the price under spot selling. From Lemmas 2 and 5 , it follows that the date- 1 APD price is always equal to the spot-selling price if the optimal spot-selling price satisfies $p^{s} \geq \bar{r}+\alpha_{L}$. This implies that consumers are better off if the monopolist uses the optimal APD policy rather than the optimal spot-selling policy because some consumers prefer to buy at a discount under APD.

Note that the conditions $c-\alpha_{H}>\underline{r}-\phi$ and $p^{s} \geq \bar{r}+\alpha_{L}$ hold for marginal costs sufficiently large. In general, however, the welfare effects of prohibiting APD policies are ambiguous. This may not be too surprising in light of the fact that the welfare effects of second-degree price 
discrimination are generally ambiguous. For instance, to compare APD with advance selling, consider the case $c<\underline{r}-\underline{\phi}$ In that case, $\widehat{p}_{0}>p^{a}=\underline{r}$. Since also $\widehat{p}_{1}=\underline{r}+\alpha_{H}=p^{a}-\alpha_{H}$, it is straightforward to see that all consumers are worse or equally well off under APD than under advance selling. As to comparing APD with spot selling, it is possible to show that, if $p^{s}<\bar{r}+\alpha_{L}$, at least some consumers are worse off when the monopolist chooses an APD policy.

\section{A Mechanism Design Perspective}

In this section, we extend the model in two directions. First, we allow for a more general shock structure in which the shock $v_{z}(r)-r$ can depend (in an affine-linear fashion) on the consumer's type $r$. Second, we consider gradual resolution of uncertainty such that the consumer obtains an informative signal about his ex-post valuation at an intermediate date $\tau$. We analyze the extended model by adopting a mechanism design approach. Most importantly, we show that the optimal mechanism can always be implemented by one of our three simple pricing policies - APD, advance selling, spot selling - and provide necessary and sufficient conditions for the optimality of each of these policies. In particular, it is not optimal for the monopolist to sell the good to some consumers at the intermediate date $\tau$.

\subsection{Model Extension}

In the baseline model, we assumed $v_{z}(r)=r+\alpha_{z}$ for $z \in\{L, H\}$ and all $r$, i.e., the shock $v_{z}(r)-r$ is independent of $r$. We now generalize by assuming that the shock is an affine-linear function of $r$ :

$$
v_{z}(r)=\alpha_{z}+\beta_{z} r, z \in\{L, H\} .
$$

Since $\mathbf{E}[v(r) \mid r]=r$, we have $\lambda_{L} \beta_{L}+\lambda_{H} \beta_{H}=1$ and $\lambda_{L} \alpha_{L}+\lambda_{H} \alpha_{H}=0$. The retained assumption that $v_{H}(r)>v_{L}(r) \geq 0$ for all $r \in[\underline{r}, \bar{r}]$ imposes additional restrictions on the values of the $\alpha_{z}$ 's and $\beta_{z}$ 's. ${ }^{8}$

In the baseline model, we assumed that there are only two selling dates: $t=0$, where each consumer only knows his type (expected valuation) $r$, and $t=1$, where each consumer knows his ex-post valuation $v(r)$. We now extend this model by assuming that each consumer obtains an informative signal $s \in\{l, h\}$ about his ex-post valuation $v(r)$ at some intermediate date $\tau \in(0,1)$. The question of interest is whether the monopolist optimally sells the good to some consumers at that date. State $s=h$ occurs with probability $\rho_{h}$ and state $s=l$ with probability $\rho_{l}$. Conditional on obtaining the signal $s$, the probability that consumer $r$ 's ex-post valuation is $v_{z}(r)$ is denoted $\lambda_{z}^{s} \in(0,1)$, where $\lambda_{L}^{s}=1-\lambda_{H}^{s}$ by construction and $\lambda_{H}^{h}>\lambda_{H}^{l}$ by the informativeness of the signal, and so $\lambda_{L}^{h}<\lambda_{L}^{l}$. Moreover, $\Sigma_{s \in\{l, h\}} \lambda_{z}^{s} \rho_{s} \equiv \lambda_{z}$ for $z \in\{L, H\}$.

\footnotetext{
${ }^{8}$ In the baseline model, $v_{H}(r)>v_{L}(r) \geq 0$ for all $r$ and $\lambda_{L} \alpha_{L}+\lambda_{H} \alpha_{H}=0$ implied $\alpha_{H}>0>\alpha_{L}$. This is no longer the case here.
} 


\subsection{A Mechanism Design Analysis}

We now adopt a mechanism design approach to analyze the extended model. First, we describe a set of direct mechanisms which we obtain when we impose only a part of the relevant incentive compatibility constraints and the ex-ante individual rationality constraint. Then, we use this larger set of direct mechanisms (which contains all incentive compatible mechanisms and some that are not incentive compatible) to derive an upper bound on the profit attained by the optimal incentive compatible mechanism. In the next subsection, we show that this upper bound on profit can always be attained by one of the three simple pricing policies (APD, advance selling, spot selling) that do not involve selling at the intermediate date $\tau$.

Since consumers obtain information sequentially, we consider the following (direct) sequential mechanism. ${ }^{9}$ Each consumer (agent) announces his type $\widehat{r} \in[\underline{r}, \bar{r}]$ at date 0 , his signal $\widehat{s} \in\{l, h\}$ at date $\tau$, and the state of his ex-post valuation $\widehat{z} \in\{L, H\}$ at date 1 . After observing $r$, the agent has, at date 0 , the option not to play the mechanism and to obtain an outside value of zero (individual rationality). After date 1 , the consumer is allocated the good with probability $q(\widehat{r}, \widehat{s}, \widehat{z})$ and has to make a payment $t(\widehat{r}, \widehat{s}, \widehat{z})$ to the monopolist (principal). A consumer's (pure) strategy is a collection of functions $\widehat{r}(r) \in[\underline{r}, \bar{r}], \widehat{s}(\widehat{r} \mid r, s) \in\{l, h\}$ and $\widehat{z}(\widehat{r}, \widehat{s} \mid r, s, z) \in\{L, H\}$ describing how previous announcements and information map into announcements. Prior to date 0 , the monopolist commits to a mechanism $\{q(\widehat{r}, \widehat{s}, \widehat{z}), t(\widehat{r}, \widehat{s}, \widehat{z})\}$. A consumer's realized net surplus is $u=q v-t$; the monopolist's profit from this consumer is $\pi=t-q c$.

We now focus on mechanisms that satisfy the incentive-compatibility constraints at date 0 only, ignoring the constraints at dates $\tau$ and 1 . The date- 0 incentive-compatibility constraints are given by

$$
\mathbf{E}[q(r, s, z) v-t(r, s, z) \mid r] \geq \mathbf{E}[q(\widehat{r}(\cdot), \widehat{s}(\cdot), \widehat{z}(\cdot)) v-t(\widehat{r}(\cdot), \widehat{s}(\cdot), \widehat{z}(\cdot)) \mid r]
$$

for any $r$ and any functions $\widehat{r}(r), \widehat{s}(\widehat{r} \mid r, s), \widehat{z}(\widehat{r}, \widehat{s} \mid r, s, z)$. Necessary for (15) is that the inequality holds for the specific functions $\widehat{s}(\widehat{r} \mid r, s)=s$ and $\widehat{z}(\widehat{r}, \widehat{s} \mid r, s, z)=z$, i.e., in the case where the agent always reveals the information he obtains at dates $\tau$ and 1 truthfully,

$$
\mathbf{E}[q(r, s, z) v-t(r, s, z) \mid r] \geq \mathbf{E}[q(\widehat{r}, s, z) v-t(\widehat{r}, s, z) \mid r]
$$

for any $r$ and any $\widehat{r}$. Eq. (16) can be written as

$$
\sum_{\substack{(s, z) \\ \in\{l, h\} \times\{L, H\}}} \rho_{s} \lambda_{z}^{s}\left[q(r, s, z) v_{z}(r)-t(r, s, z)\right] \geq \sum_{\substack{(s, z) \\ \in\{l, h\} \times\{L, H\}}} \rho_{s} \lambda_{z}^{s}\left[q(\widehat{r}, s, z) v_{z}(r)-t(\widehat{r}, s, z)\right] .
$$

\footnotetext{
${ }^{9}$ Since the revelation priniciple applies, we can restrict attention to direct mechanisms without loss of generality.
} 
Define

$$
\begin{aligned}
Q(r) & \equiv \sum_{\substack{(s, z) \\
\epsilon\{l, h\} \times\{L, H\}}} \rho_{s} \lambda_{z}^{s} \beta_{z} q(r, s, z) \\
S(r) & \equiv \sum_{\substack{(s, z) \\
\epsilon\{l, h\} \times\{L, H\}}} \rho_{s} \lambda_{z}^{s}\left[t(r, s, z)-\alpha_{z} q(r, s, z)\right] \text { and } \\
T(r) & \equiv \sum_{\substack{(s, z) \\
\epsilon\{l, h\} \times\{L, H\}}} \rho_{s} \lambda_{z}^{s} t(r, s, z) .
\end{aligned}
$$

Then, using the linear structure of $v_{z}(r)$, Eq. (17) becomes

$$
Q(r) r-S(r) \geq Q(\widehat{r}) r-S(\widehat{r}) .
$$

After interchanging the roles of $r$ and $\widehat{r}$, we obtain

$$
Q(\widehat{r}) \widehat{r}-S(\widehat{r}) \geq Q(r) \widehat{r}-S(r) .
$$

Note that (18) and (19) together imply

$$
[Q(r)-Q(\widehat{r})] r \geq S(r)-S(\widehat{r}) \geq[Q(r)-Q(\widehat{r})] \widehat{r}
$$

which can be satisfied only if $Q(r)$ is weakly increasing. Monotonicity of $Q(r)$ implies that $Q(r)$ must be differentiable almost everywhere. Moreover, $\mathbf{E}[q(r, s, z) v-t(r, s, z) \mid r]=Q(r) r-S(r)$ is continuous for any $r \in[\underline{r}, \bar{r}] .^{10}$

After dividing Eq. (20) by $r-\widehat{r}$ and letting $\widehat{r} \rightarrow r$, we obtain for almost all $r$ that $Q^{\prime}(r) r-S^{\prime}(r)=0$. After integrating (and using continuity of $Q(r) r-S(r)$ ), we get $S(r)=$ $Q(r) r-\int_{\underline{r}}^{r} Q(\rho) \mathrm{d} \rho-[Q(\underline{r}) \underline{r}-S(\underline{r})]$. Rewriting yields the following condition on the expected transfer:

$$
\begin{aligned}
T(r)= & \sum_{\substack{(s, z) \\
\in\{l, h\} \times\{L, H\}}} \rho_{s} \lambda_{z}^{s} q(r, s, z) v_{z}(r)-\int_{\underline{r}}^{r} \sum_{\substack{(s, z) \\
\in\{l, h\} \times\{L, H\}}} \rho_{s} \lambda_{z}^{s} \beta_{z} q(\xi, s, z) \mathrm{d} \xi \\
& -\mathbf{E}[q(r, s, z) v-t(r, s, z) \mid r=\underline{r}] .
\end{aligned}
$$

We thus obtain the following lemma:

Lemma 7 A necessary condition for incentive compatibility is that Eq. (21) is satisfied for all $r \in[\underline{r}, \bar{r}]$.

\footnotetext{
${ }^{10}$ Assume to the contrary that $\mathbf{E}[q(r, s, z) v-t(r, s, z) \mid r]$ is not continuous. Then there exists a value $r$ and a sequence $\left\{r_{i}\right\}_{i}$ converging to $r$ such that $\lim _{i \rightarrow \infty} Q\left(r_{i}\right) r_{i}-S\left(r_{i}\right) \neq Q(r) r-S(r)$. Equation (18) implies $[Q(r) r-S(r)]-\left[Q\left(r_{i}\right) r_{i}-S\left(r_{i}\right)\right] \geq Q\left(r_{i}\right)\left(r-r_{i}\right)$ and $(19)$ implies $Q(r)\left(r-r_{i}\right) \geq[Q(r) r-S(r)]-\left[Q\left(r_{i}\right) r_{i}-\right.$ $\left.S\left(r_{i}\right)\right]$. Note that $Q(r)$ is a linear combination of the $q(r, \cdot, \cdot)$ 's (which are probabilities), with the weights being independent of $r$. Hence, $Q(\cdot)$ is bounded, and thus $\lim _{r_{i} \rightarrow r} Q\left(r_{i}\right)\left(r-r_{i}\right)=0$ and $\lim _{r_{i} \rightarrow r} Q(r)\left(r-r_{i}\right)=0$, implying $\lim _{r_{i} \rightarrow r}[Q(r) r-S(r)]-\left[Q\left(r_{i}\right) r_{i}-S\left(r_{i}\right)\right]=0$, a contradiction.
} 
We now derive an upper bound on profit attained by the optimal mechanism. To this end, we consider mechanisms with allocation rule $q(r, s, z)$ and transfer function $t(r, s, z)$ that satisfy the necessary condition for incentive compatibility from Lemma 7 and individual rationality.

The monopolist's expected profit is

$$
\begin{aligned}
\Pi \equiv & \int_{\underline{r}}^{\bar{r}}\left[T(r)-\sum_{\substack{(s, z) \\
\in\{l, h\} \times\{L, H\}}} \rho_{s} \lambda_{z}^{s} q(r, s, z) c\right] f(r) \mathrm{d} r \\
= & \int_{\underline{r}}^{\bar{r}}\left[\sum_{\substack{(s, z) \\
\in\{l, h\} \times\{L, H\}}} \rho_{s} \lambda_{z}^{s} q(r, s, z)\left[v_{z}(r)-c\right]-\int_{\underline{r}}^{r} \sum_{\substack{(s, z) \\
\in\{l, h\} \times\{L, H\}}} \rho_{s} \lambda_{z}^{s} \beta_{z} q(\rho, s, z) \mathrm{d} \rho\right] f(r) \mathrm{d} r \\
& -\mathbf{E}[q(r, s, z) v-t(r, s, z) \mid r=\underline{r}] .
\end{aligned}
$$

Note that the integral on the RHS of (22) exists as the distribution of $r$ has a finite mean. Integrating by parts, Eq. (22) can be written as

$$
\begin{aligned}
\Pi= & \int_{\underline{r}}^{\bar{r}} \sum_{\substack{(s, z) \\
\in\{l, h\} \times\{L, H\}}} \rho_{s} \lambda_{z}^{s} q(r, s, z)\left[v_{z}(r)-c-\frac{1-F(r)}{f(r)}\right] f(r) \mathrm{d} r \\
& -\mathbf{E}[q(r, s, z) v-t(r, s, z) \mid r=\underline{r}] .
\end{aligned}
$$

By individual rationality, $\mathbf{E}[q(r, s, z) v-t(r, s, z) \mid r=\underline{r}] \geq 0$. Letting

$$
w_{z}(r) \equiv v_{z}(r)-c-\frac{1-F(r)}{f(r)}
$$

with $w_{z}(\underline{r})=v_{z}(\underline{r})-c-\underline{\phi}$ and $w_{z}(\bar{r})=v_{z}(\bar{r})-c-\bar{\phi}$, we obtain an upper bound on profit:

$$
\begin{aligned}
\Pi & \leq \int_{\underline{r}}^{\bar{r}} \sum_{\substack{(s, z) \\
\in\{l, h\} \times\{L, H\}}} \rho_{s} \lambda_{z}^{s} q(r, s, z) w_{z}(r) f(r) \mathrm{d} r \\
& \leq \int_{\underline{r}}^{\bar{r}} \sum_{\substack{(s, z) \\
\in\{l, h\} \times\{L, H\}}} \rho_{s} \lambda_{z}^{s} \max \left\{w_{z}(r), 0\right\} f(r) \mathrm{d} r \equiv \bar{\Pi} .
\end{aligned}
$$

We thus have derived the following lemma:

Lemma 8 The profit attained by the optimal mechanism cannot exceed $\bar{\Pi}$, as defined in Eq. (24).

From (23) and (24), we obtain the following proposition. 
Proposition 3 If there exists an incentive-compatible mechanism for which

$$
q(r, s, z)\left\{\begin{array}{cl}
=1 & \text { if } w_{z}(r)>0 \\
\in[0,1] & \text { if } w_{z}(r)=0 \\
=0 & \text { if } w_{z}(r)<0
\end{array}\right.
$$

and

$$
\mathbf{E}[q(r, s, z) v-t(r, s, z) \mid r=\underline{r}]=0,
$$

then this mechanism maximizes the monopolist's profit. Moreover, if an incentive-compatible mechanism satisfying Eqs. (25) and (26) exists, then any optimal (profit-maximizing) mechanism must satisfy these equations (Eq. (25) almost everywhere).

\subsection{Implementing the Optimal Mechanism through Simple Pricing Policies}

Assuming that $w_{z}(r)$ is strictly increasing in $r$ for $z \in\{L, H\}$, which is implied by $\beta_{z} \geq 0$, we now show that the upper bound on profit can always be obtained by a simple pricing policy $\left(p_{0}, p_{\tau}, p_{1}\right)$ that does not involve selling at the intermediate date $\tau$. We distinguish three cases.

Case 1: $w_{L}(\underline{r}) \geq 0$. In this case, $w_{z}(r) \geq 0$ for all $r, s$, and $z$. Hence, the monopolist always wants to sell to all consumers. Setting the probability $q(r, s, z)=1$ and the transfer $t(r, s, z)=\mathbf{E}[q(r, s, z) v \mid r=\underline{r}]$ is an incentive-compatible mechanism that satisfies Eqs. (25) and (26). Note that the mechanism is constant, i.e., $q(r, s, z)$ and $t(r, s, z)$ do not depend on $r$, $s$, and $z$. Advance selling at price $p_{0}=\underline{r}$ implements this mechanism (and trivially satisfies the neglected incentive compatibility constraints) while other simple selling policies do not and are therefore not optimal. For instance, for spot selling to yield $q(r, s, z)=1$ for all $r, s$, and $z$, the spot-selling price would have to be set at $p_{1}=v_{L}(\underline{r})<\underline{r}$, i.e., $t(r, s, z)=v_{L}(\underline{r})$, implying that the date-0 individual rationality constraint is slack even for the lowest type. But this violates Eq. (26).

Case 2: $w_{L}(\bar{r}) \leq 0$. In this case, $w_{L}(r) \leq 0$ for all $r$ and $s$. Hence, the monopolist never wants to sell to consumers who turn out to be in state $z=L$, i.e., $q(r, s, L)=0$ and $t(r, s, L)=0$ for all $r$ and $s$. However, the monopolist wants to sell with probability one to those consumers who turn out to be in state $z=H$ and who are of sufficiently high type $r \geq \widetilde{r} \equiv \min \left\{r \mid w_{H}(r) \geq 0\right\}$, and never to consumers with $r<\widetilde{r}$ :

$$
q(r, s, H)=\left\{\begin{array}{ll}
1 & \text { if } r \geq \widetilde{r} \\
0 & \text { if } r<\widetilde{r}
\end{array} .\right.
$$

Inserting these probabilities into Eq. (21) yields the transfer payments:

$$
t(r, s, H)=\left\{\begin{array}{cl}
v_{H}(\widetilde{r}) & \text { if } r \geq \widetilde{r} \\
0 & \text { if } r<\widetilde{r}
\end{array} .\right.
$$

It can easily be checked that this mechanism satisfies Eqs. (25) and (26). Spot selling with date1 price $p_{1}=v_{H}(\widetilde{r})$ implements this mechanism (and trivially satisfies the neglected incentive compatibility constraints), while other simple pricing policies do not and are therefore not optimal. 
Case 3. $w_{L}(\underline{r})<0$ and $w_{L}(\bar{r})>0$. In this case, the monopolist wants to sell to the highest types with probability one, independently of the realization of $s$ and $z$, never to the lowest types, and to intermediate types only in state $z=H$, independently of the realization of $s$ :

$$
\begin{gathered}
q(r, s, L)=\left\{\begin{array}{ll}
1 & \text { if } r \geq \widetilde{r}^{\prime \prime} \\
0 & \text { if } r<\widetilde{r}^{\prime \prime}
\end{array},\right. \\
q(r, s, H)=\left\{\begin{array}{ll}
1 & \text { if } r \geq \widetilde{r}^{\prime} \\
0 & \text { if } r<\widetilde{r}^{\prime}
\end{array},\right.
\end{gathered}
$$

where $\widetilde{r}^{\prime} \equiv \min \left\{r \mid w_{H}(r) \geq 0\right\}$ and $\widetilde{r}^{\prime \prime} \equiv \min \left\{r \mid w_{L}(r) \geq 0\right\}$. Since $w_{H}(r)>w_{L}(r)$, we have $\widetilde{r}^{\prime}<\widetilde{r}^{\prime \prime}$. Inserting these probabilities into Eq. (21) yields the transfer payments:

$$
\begin{aligned}
& t(r, s, L)=\left\{\begin{array}{cl}
\lambda_{H} v_{H}\left(\widetilde{r}^{\prime}\right)+\lambda_{L} v_{L}\left(\widetilde{r}^{\prime \prime}\right) & \text { if } r \geq \widetilde{r}^{\prime \prime} \\
0 & \text { if } r<\widetilde{r}^{\prime \prime}
\end{array},\right. \\
& t(r, s, H)=\left\{\begin{array}{cl}
\lambda_{H} v_{H}\left(\widetilde{r}^{\prime}\right)+\lambda_{L} v_{L}\left(\widetilde{r}^{\prime \prime}\right) & \text { if } r \geq \widetilde{r}^{\prime \prime} \\
v_{H}\left(\widetilde{r}^{\prime}\right) & \text { if } \widetilde{r}^{\prime} \leq r<\widetilde{r}^{\prime \prime} \\
0 & \text { if } r<\widetilde{r}^{\prime}
\end{array}\right.
\end{aligned}
$$

It can easily be checked that this mechanism satisfies Eqs. (25) and (26). An APD policy with a price path given by Lemma 2 implements this mechanism and also satisfies the neglected incentive compatibility constraints. Again, it is straightforward to verify that other simple pricing policies do not implement the optimal mechanism.

These insights are summarized in the following proposition:

Proposition 4 Suppose that $w_{z}(r)$ is strictly increasing in $r$ for $z \in\{L, H\}$. The optimal mechanism can always be implemented by a simple pricing policy with sales at dates 0 or 1 only. Specifically, the optimal mechanism is implemented by:

1. an advance-selling policy if $w_{L}(\underline{r}) \geq 0$,

2. a spot-selling policy if $w_{L}(\bar{r}) \leq 0$, and

3. an APD policy otherwise.

Spot selling and advance selling emerge only as special cases of the optimal mechanism. The "normal" case is the one where an APD policy is optimal. To see this, note that $w_{L}(\underline{r})<0<$ $w_{L}(\bar{r})$ if the support of $r$ is sufficiently large (in that $\underline{r}$ is sufficiently small and $\bar{r}$ sufficiently large) and $\beta_{L}>0$. However, if all consumers have (almost) the same ex-post valuation conditional on suffering a negative shock $z=L$, and this valuation is less than the marginal cost $c$, then spot selling implements the optimal mechanism: Assuming $\alpha_{L}<c$, we have $w_{L}(\bar{r})<0$ for $\beta_{L}$ sufficiently small.

What is the intuition for our main result? By Proposition 3, any mechanism that induces a profit level equal to the upper bound $\bar{\Pi}$ has the following two properties: it gives zero expected utility to a consumer of type $r=\underline{r}$ and is characterized by two thresholds in the type space, 
$\widetilde{r}^{\prime}=w_{H}^{-1}(0)$ and $\widetilde{r}^{\prime \prime}=w_{L}^{-1}(0)$, with $r^{\prime}<r^{\prime \prime}$. If $r \in\left[\widetilde{r}^{\prime \prime}, \bar{r}\right]$, the consumer should always obtain the good (condition I). If $r \in\left[\widetilde{r}^{\prime}, \widetilde{r}^{\prime \prime}\right)$, the consumer should obtain the good only in the event $z=H$ (condition II). Whether condition I is met depends only on the consumer's date- 0 information. Whether condition II is met can be decided only after the consumer has learned his date- 1 information. The monopolist's objective is, thus, to sell to the consumer at date 1 if condition II is satisfied, and at date 0 if condition I is satisfied. It is always possible to achieve this objective through a simple pricing policy: Suppose that the monopolist faces at date 1 only those consumers with $r \in\left[\underline{r}, \widetilde{r}^{\prime \prime}\right)$ because consumers with $r \geq \widetilde{r}^{\prime \prime}$ already obtained the object at date 0 . Condition II is satisfied if and only if the consumer's realized valuation $v_{z}(r)$ is larger than $v_{1} \equiv c+\left(1-F\left(\widetilde{r}^{\prime}\right)\right) / f\left(\widetilde{r}^{\prime}\right) .{ }^{11}$ Hence, the monopolist needs to screen only the consumer's realized valuation. This can be achieved by offering the consumer the option to buy the good at date 1 for a fixed price $v_{1}$. It follows that a consumer with $r \leq \widetilde{r}^{\prime}$ obtains zero expected utility. It remains to be verified that consumers with $r \geq \widetilde{r}^{\prime \prime}$ can be screened at date 0 . Since $\mathbf{E}[v(r) \mid r]=r$, this amounts to screening expected date- 0 valuations. As a consumer with $r \geq \widetilde{r}^{\prime \prime}$ has a positive option value of postponing his purchasing decision until date $1, \lambda_{H}\left(v_{H}(r)-v_{1}\right)$, the monopolist has to grant him a discount in order to incentivize him to buy already at date 0 . The desired screening level is obtained by offering him the option to buy at date 0 at price $\widetilde{r}^{\prime \prime}-\lambda_{H}\left(v_{H}\left(\widetilde{r}^{\prime}\right)-v_{1}\right)=\lambda_{H} v_{H}\left(\widetilde{r}^{\prime}\right)+\lambda_{L} v_{L}\left(\widetilde{r}^{\prime \prime}\right) .{ }^{12}$

We conclude this section by highlighting some properties and extensions which build upon this intuition:

Remark 1 Why can the private information $z$ be handled as if it were public? In the proof of Proposition 3, we consider the case where the realization of $z$ is public information, although we are interested in the case where it is private information to the consumer. We use this benchmark case to derive an upper bound on welfare. In Proposition 4 we then show that there exists a mechanism which attains this upper bound. In this mechanism, a consumer with $z=L$ does not buy the good at the date-1 price. It follows that it cannot be optimal for a consumer with $z=H$ to mimic, at date 1 , a consumer with $z=L$. As a consequence, the monopolist can elicit $z$ without having to pay an information rent to the consumer. While the monopolist's desired allocation depends on $z$, the constructed sequential screening mechanism allows the monopolist to obtain the private information $z$ at date 1 "for free".

Remark 2 Note that the logic of the proof of Proposition 3 does not rely on the specific structure of the information at an intermediate date $\tau$. For instance, it might be continuous or non-binary. Any kind of information at the intermediate date attains the same upper bound on welfare as derived in the proof of Proposition 3. Moreover, if this upper bound is attainable by some mechanism which does not make use of this information, the corresponding mechanism

\footnotetext{
${ }^{11}$ Equilibrium requires $w_{H}\left(\widetilde{r}^{\prime}\right)=0$, implying $v_{H}\left(\widetilde{r}^{\prime}\right)=c+\left(1-F\left(\widetilde{r}^{\prime}\right)\right) / f\left(\widetilde{r}^{\prime}\right)$. As $v_{H}(\cdot)$ is strictly increasing, the value of an agent with $z=H$ is larger than $v_{1}=v_{H}\left(\widetilde{r}^{\prime}\right)$ if $r \geq \widetilde{r}^{\prime}$. Moreover, since equilibrium requires $w_{L}\left(\widetilde{r}^{\prime \prime}\right)=0$, it follows that $v_{L}\left(\widetilde{r}^{\prime \prime}\right)=c+\left(1-F\left(\widetilde{r}^{\prime \prime}\right)\right) / f\left(\widetilde{r}^{\prime \prime}\right)<c+\left(1-F\left(\widetilde{r}^{\prime}\right)\right) / \widetilde{r}^{\prime}=v_{1}$. As $v_{L}(\cdot)$ is strictly increasing, we have $v_{L}(r)<v_{1}$ for any $r \in\left[\underline{r}, \widetilde{r}^{\prime \prime}\right)$.

${ }^{12}$ If $r=\widetilde{r}^{\prime \prime}$, the agent just pays his (expected) valuation minus his option value. If $r>r^{\prime \prime}$, he is strictly better off buying early.
} 
must be optimal for any such information. It follows that, under the conditions of Proposition 4, the specific structure of the information at date $\tau$ does not matter for the result.

Remark 3 Why is there no trade at the intermediate date $\tau$ ? This is for the same reason why the monopolist does not want to use a stochastic mechanism in the two-period model (without any information at an intermediate date). In the proof of Proposition 3, we assume that the monopolist learns the realized $z$ for each consumer and we derive the optimal mechanism under this assumption. In the two-period model, she could construct a random variable s, correlated with z. Similarly, in the extended model with gradual resolution of uncertainty, the monopolist could use any given random variable $s$, correlated with $z$. Since she has no incentive to use the random variable $s$ in the former case, she has no incentive to use it in the latter.

Remark 4 Do our optimality results require binary date-1 information? If date-1 information is non-binary, it is typically no longer the case that that the monopolist needs to screen only valuations at date 1. By proceeding in an analogous fashion to Proposition 3, we can derive an allocation that is optimal if it is also implementable in the case where $z$ is private information. In contrast to the case of binary information at date 1, with non-binary information, it may happen that, for the same value of $v_{z}(r)$, the monopolist desires different allocations. More precisely, there may exist consumers $\left(r_{1}, z_{1}\right)$ and $\left(r_{2}, z_{2}\right)$ such that $v_{z_{1}}\left(r_{1}\right)=v_{z_{2}}\left(r_{2}\right)$, but where the good is optimally allocated to only one of them. ${ }^{13}$ However, since the consumer's utility depends on his information only indirectly through his valuation, he will for the same valuations undertake the same decisions (unless he is indifferent). Since simple pricing policies are only capable of implementing allocations where the same valuations lead to the same decisions, they are (in general) not capable of implementing the reference allocation. ${ }^{14}$ Hence, our strategy of proof does not work for the non-binary case since date-1 information can no longer be handled as if it were public. The monopolist may want to use "more" of the date-0 information at later dates (for instance, through option contracts) and she may want to use "less" date-1 information to reduce information rents. Moreover, in the case with gradual resolution of uncertainty, trading at intermediate dates or conditioning on intermediate information may become beneficial.

\footnotetext{
${ }^{13}$ As an example, consider the case where $z$ can assume three values $L, M$ and $H$. Moreover, suppose that $c=0, r \sim U[0,1], \lambda_{L}=\lambda_{M}=\lambda_{H}=1 / 3, \alpha_{L}=\alpha_{M}=\alpha_{H}=0, \beta_{L}=1 / 2, \beta_{M}=1$ and $\beta_{H}=3 / 2$. Then the monopolist wants to implement the following allocation: If $r \in[0,0.4)$, the agent does not obtain the good; if $r \in[0.4,0.5)$, he obtains the object only if $z=H$; if $r \in[0.5,2 / 3)$, he obtains the good if only if $z=H$ or $z=M$; and if $z \in[2 / 3,1]$, he always obtains the object. Let $r_{1} \in(1 / 3,0.4)$ and $r_{2}\left(r_{1}\right)=3 / 2 r_{1}$, so that $v_{H}\left(r_{1}\right)=v_{M}\left(r_{2}\right)$. Although both consumers $\left(r_{1}, H\right)$ and $\left(r_{2}, M\right)$ have the same ex-post valuation, the optimal allocation is such that the good is allocated only to $\left(r_{2}, M\right)$.

${ }^{14}$ There exist some special cases where simple pricing policies are still capable of implementing the reference allocation: (i) If we adjust the example in Footnote 13 by defining $\beta_{M} \equiv 1+x$ and $\beta_{H} \equiv 1 / 2-x$ with $x \in(0,1 / 2)$, then there exists a single $x$-value, $x=1 / 5$, for which the monopolist needs to screen only valuations at date 1 . Hence, in this case, there exists a simple pricing policy which is capable of implementing the reference allocation. (ii) For specific forms of gradual information revelation, simple pricing policies with trade at more than two dates may be capable of implementing the reference allocation. As an example, consider again the case where $z$ can assume three values, as described in Footnote 13. If the consumer learns at an intermediate date whether $z=L$ or $z \neq L$, the construction for the binary case can be "applied iteratively". This way, a simple pricing policy is constructed where consumers buy at date 0 if $r \in[2 / 3,1]$ and $z \in\{L, M, H\}$; at date $\tau$ if $r \in[0.5,2 / 3)$ and $z \in\{M, H\}$; and at date 1 if $r \in[0.4,0.5)$ and $r=H$.
} 
Remark 5 Although we assumed that there is only an individual rationality constraint at date 0 , there always exists an implementation of the optimal mechanism such that giving the consumer also an outside option of zero at dates $\tau$ and 1 does not alter the conclusion. One such implementation is the optimal simple pricing policy.

Remark 6 From the fact that simple pricing policies strictly dominate stochastic mechanisms, it follows that the monopolist does not want to ration consumers by committing to a price-quantity path $\left(p_{0}, q_{0} ; p_{1}, q_{1}\right)$ if such rationing is inefficient (in the sense that higher types are rationed with a probability strictly between zero and one, as in the case of proportional rationing). Moreover, as we now argue, the monopolist does not want to ration consumers if the rationing rule is efficient. To see this, suppose first that the monopolist rations consumers at date 0. Consider the following deviation: the monopolist slightly increases price $p_{0}$, keeping quantity $q_{0}$ fixed. Since this will leave unchanged the set of consumers that buy at either date, the deviation increases the monopolist's date-0 profit without affecting her date-1 profit. Suppose second that the monopolist rations consumers at date 1. Consider the following deviation: The monopolist slightly increases quantity $q_{1}$, keeping price $p_{1}$ fixed. Under efficient rationing, this will not affect the set of consumers that buy at date 0 (as these are high types who would not be rationed at date 1) and, hence, will not affect the monopolist's date-0 profit but increases her date-1 profit. To summarize, the optimal simple pricing policy strictly dominates any price-quantity path $\left(p_{0}, q_{0} ; p_{1}, q_{1}\right)$ that induces consumer rationing, independently of the rationing rule.

Remark 7 The above analysis relies on the assumption that $\beta_{z} \geq 0$ for $z \in\{L, H\}$. A rather different result obtains when $\beta_{L}<0<\beta_{H}$. Suppose that $w_{L}(r)$ is decreasing in $r$. (Indeed, if the hazard rate of $r$ is finite everywhere, then $w_{L}(r)$ is decreasing in $r$ for $\beta_{L}$ sufficiently negative. $)^{15}$ Then, an APD policy implements the optimal mechanism if and only if $w_{L}(\underline{r})>0>w_{L}(\bar{r})$. In contrast to the APD policy considered above, however, it will be the low types that buy the good at $t=0$ and the high types that buy the good at $t=1$ whenever $z=H$. To understand this, note that the high types face much greater uncertainty than the low types when $w_{L}(r)$ is decreasing in $r$. The optimal mechanism, in this case, prescribes selling to the high types only when $z=H$ and selling to the low types independently of $z$. (The same result would obtain if consumers differed only in the degree of uncertainty they face but not in their expected valuations.)

\section{Conclusion}

In this paper, we have provided a novel theory of advance-purchase discounts in which advancepurchase discounts serve as a pure price discrimination device. In contrast to existing explanations of advance-purchase discounts, our theory does not rely on scarce capacity or aggregate demand uncertainty. The key feature of our theory is that consumers face individual uncertainty over their future valuation for the good and this uncertainty is resolved over time. This

\footnotetext{
${ }^{15}$ Otherwise, if $w_{L}(r)$ is not monotonic in $r$, the optimal mechanism may not be implementable through simple pricing policies. Note that $w_{L}(r)$ is always monotonic in $r$ if $F$ is uniform.
} 
allows a monopoly seller to charge different prices for the same product at different dates prior to consumption. Consumers with a high expected valuation will purchase the product in advance at a discount, while consumers with a low expected valuation will delay their purchasing decision and buy at the regular price only when their realized valuation turns out to be high. Assuming that the distribution of shocks to consumer preferences is binary, we obtain a necessary and sufficient condition under which advance-purchase discounts implement the monopolist's optimal mechanism. This implies, in particular, that more complicated contracts such as partial refund contracts cannot lead to higher profits.

Our analysis has been motivated by the pricing policies for holiday packages, hotel rooms, rental car hires, and conferences. Another group of examples are tickets for the soccer world cup and other sports or cultural events. For instance, consider the ticket sale for a particular match. Consumers are willing to pay a premium in the event that their favorite team or player makes it into this match. Thus, the organizers of the tournament can use advance-purchase discounts as a price discrimination strategy (provided secondary markets can be dried out, which may be achieved by "personalizing" tickets as in the 2006 soccer world cup). Relatedly, active participation in a sports event (such as a mass running event) typically requires personal registration. Our analysis also extends to the introduction of new experience goods where consumers face uncertainty about the product's characteristics. For our theory to be directly applicable, this uncertainty must reflect horizontal taste heterogeneity. In such a setting, consumers with a high expected valuation tend to buy early, while those with a lower expected valuation wait and buy only if the actual product characteristics fit their taste. ${ }^{16}$

\footnotetext{
${ }^{16}$ An example is the practice by premium vineries to post subscription prices for wine; that is, consumers are offered to buy the vintage at a discount before it is bottled. A buyer who buys such a subscription cannot rely on taste tests and recommendations since the product does not yet exist.
} 


\section{References}

[1] M. Bagnoli, T. Bergstrom, Log Concave Probability and its Applications, Economic Theory 26 (2005), 445-469.

[2] H. Bar-Isaac, G. Caruana, V. Cunat, Information Gathering and Marketing, Journal of Economics and Management Strategy 19 (2010), 375-401

[3] P. Courty, Ticket Pricing under Demand Uncertainty, Journal of Law and Economics 46 (2003), 627-652.

[4] P. Courty, H. Li, Sequential Screening, Review of Economic Studies 67 (2000), 697-717.

[5] J. Cremer, F. Khalil, Gathering Information before Signing a Contract, American Economic Review 82 (1992), 566-578.

[6] J. Dana, Advance-Purchase Discounts and Price Discrimination in Competitive Markets, Journal of Political Economy 106 (1998), 395-422.

[7] J. Dana, Using Yield Management to Shift Demand When the Peak Time is Unknown, Rand Journal of Economics 30 (1999), 456-474.

[8] J. Dana, Equilibrium Price Dispersion under Demand Uncertainty: The Roles of Costly Capacity and Market Structure, Rand Journal of Economics 30 (1999), 632-660.

[9] J. Dana, Monopoly Price Dispersion Under Demand Uncertainty, International Economic Review 42 (2001), 649-670.

[10] I. Gale, T. Holmes, The Efficiency of Advance-Purchase Discounts in the Presence of Aggregate Demand Uncertainty, International Journal of Industrial Organization 10 (1992), 413-37.

[11] I. Gale, T. Holmes, Advance-Purchase Discounts and Monopoly Allocation of Capacity, American Economic Review 83 (1993), 135-146.

[12] T. Lewis, D. Sappington, Supplying Information to Facilitate Price Discrimination, International Economic Review 95 (1994), 309-327.

[13] R. P. McAfee, J. McMillan, M. Whinston, Multiproduct Monopoly, Commodity Bundling and Correlation of Values, Quarterly Journal of Economics 104 (1989), 371-384.

[14] M. Möller, M. Watanabe, Advance Purchase Discounts versus Clearance Sales, Economic Journal, in press.

[15] J. Riley, R. Zeckhauser, Optimal Selling Strategies: When to Haggle, When to Hold Firm, Quarterly Journal of Economics 98 (1983), 267-289.

[16] C. Wilson, The Optimal Pricing Policy of a Monopolist, Journal of Political Economy 96 (1988), 164-176. 